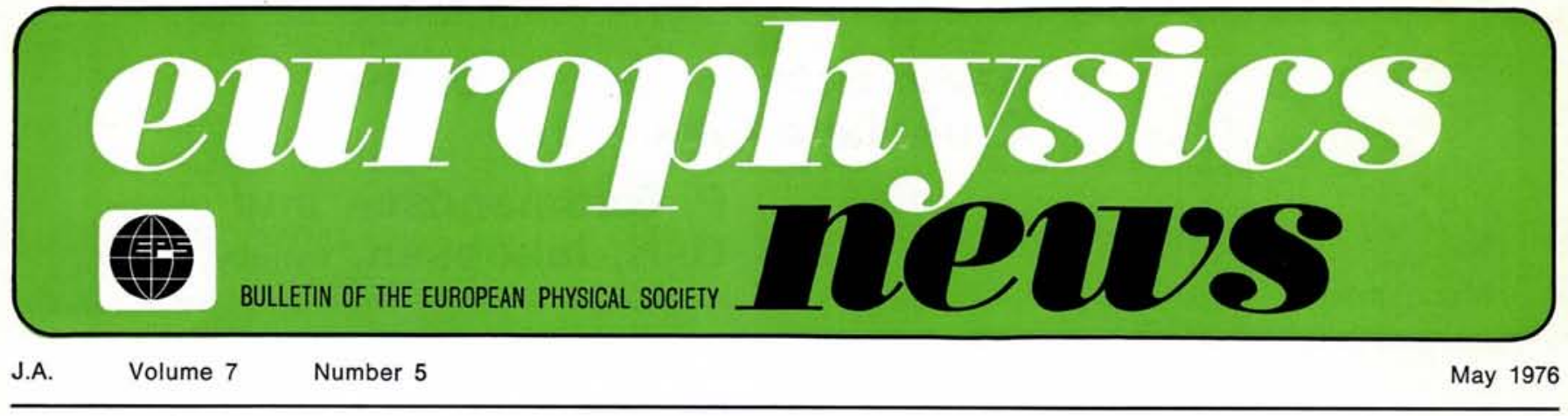

\title{
Collaboration in Physics within the Nordic Countries
}

\author{
H. H. Jensen, Copenhagen \\ (University of Copenhagen)
}

A long tradition of cultural and, more specifically, scientific collaboration exists between the five Nordic countries Sweden, Norway, Iceland, Finland and Denmark. Over the past 20 years in particular, such collaboration has had strong political support from parliaments and governments, and a large part of it was brought into a formal framework by the signing in 1971 of a government agreement. One of the provisions of this agreement is the establishment of a permanent Secretariat for Nordic Cultural Collaboration with a number of committees, of which the advisory committee on research is the one relating closest to the work of the physicists. Most of the Nordic collaboration in the field of physics is coordinated, evaluated and financed either through this Secretariat or by the national research councils, acting through a joint Nordic Committee of collaboration.

In theoretical physics the cornerstone in the collaboration is the Nordic Institute for Theoretical Atomic Physics (NORDITA). It is located in Copenhagen on the same site as the Niels Bohr Institute. The close collaboration between the two institutes has been of vital importance to both. The main fields of research and teaching at NORDITA are the theoretical aspects of nuclear physics, particle physics, astrophysics and recently added, solid state physics. The institute has a permanent staff (about 10 scientists) and about 15 fellows (on stipends of two to three years duration) from the Nordic countries. Fur- ther, a substantial number of foreign guests work under the auspices of NORDITA, either at the institute in Copenhagen, at other theoretical physics institutes in the five countries or as participants in symposia arranged or supported by NORDITA.

In order to make full use of the facilities for post-graduate and postdoctoral education, a formal framework has been established under the cultural agreement for supporting Nordic symposia, summer schools and similar short courses in all fields of science.

There has always been close contact between the Nordic experimental nuclear physicists and regular exchanges of scientists. Some years ago they agreed on a plan for a joint institute with a large medium-energy accelerator. For financial reasons the plan failed. Instead a Committee for accelerator-based research was created under the cultural agreement. But its scope does not include experimental "particle physics". In this field Nordic collaboration has evolved around the research carried out at CERN in Geneva. For example a "Scandinavian Bubble-Chamber Collaboration" has existed for nearly ten years, the purpose which is to run joint programmes at CERN and to share the local facilities for reading and interpreting bubble-chamber pictures. Similarly, since 1969 a Scandinavian Intersecting Storage Ring (ISR) collaboration has been in existence for experiments with colliding beams.

In this same field should also be mentioned the so-called ISOLDE pro- ject which started as a joint Scandinavian initiative. The name stands for Isotope Separator on Line and the purpose of the programme is to carry out investigations on short lived nuclei at the CERN synchrocyclotron.

In applied physics a number of formal and informal types of collaboration exist. Quite naturally the research in geophysics is well coordinated. For example, joint organizations exist in physical oceanography and in space research, and in many cases the Nordic geophysicists have operated joint programmes in connection with international efforts.

Finally it should be mentioned that some applied physics is included in the extensive Nordic collaboration concerned with the technical sciences, e.g. between the atomic energy research establishments and within the Nordic organization for technological research (NORDFORSK).

\section{Contents \\ Collaboration in Physics within the Nordic Countries . . . \\ Radio Echo Sounding of the Greenland Inland Ice . . . Cosmical Geophysical \\ Research \\ Ionospheric Research in the European Auroral Zone by Incoherent Scatter Techniques \\ Society News \\ The Europhysics Journals . 10 \\ Council Report from Leipzig . 11 \\ Short Notes . . . . . . 12}

